\title{
Steiner Wiener Index of the Square of Graphs*
}

\author{
Daqian Chai, Xinhui An ${ }^{\dagger}$, Baoyindureng Wu \\ College of Mathematics and System Sciences, Xinjiang University, \\ Urumqi 830046, China
}

(Received May 14, 2021)

\begin{abstract}
The square of a graph $G$, denoted by $G^{2}$, is a graph with the same vertex set as $G$, in which two vertices are adjacent if and only if their distance is at most 2 in $G$. For $S \subseteq V(G)$, the Steiner distance $d(S)$ of $S$ is the minimum size of a connected subgraph of $G$ whose vertex set contains $S$. The $k$ th Steiner Wiener index $S W_{k}(G)$ of $G$ is defined as the sum of Steiner distances of all $k$-element subsets of $V(G)$. In this paper, we show that for any tree $T$ of order $n$,
\end{abstract}

$$
S W_{3}\left(S_{n}^{2}\right) \leq S W_{3}\left(T^{2}\right) \leq S W_{3}\left(P_{n}^{2}\right),
$$

where $S_{n}$ and $P_{n}$ are the star and path of the order $n$, respectively. Let $G$ be a connected graph of order $n \geq 5$ with connected complement $\bar{G}$. We establish the Nordahaus-Gaddum type result for a connected graph $G$ with connected complement $\bar{G}$ :

$$
4\left(\begin{array}{l}
n \\
3
\end{array}\right) \leq S W_{3}\left(G^{2}\right)+S W_{3}\left(\bar{G}^{2}\right) \leq S W_{3}\left(P_{n}^{2}\right)+S W_{3}\left({\overline{P_{n}}}^{2}\right)
$$

and

$$
4 \leq \operatorname{siam}_{3}\left(G^{2}\right)+\operatorname{siam}_{3}\left(\bar{G}^{2}\right) \leq \begin{cases}\left\lceil\frac{n}{2}\right\rceil+2 & \text { if } n \geq 9 \\ 6 & \text { otherwise }\end{cases}
$$

where $\operatorname{sdiam}_{3}(G)$ is Steiner 3-diameter of $G$.

\section{Introduction}

In this paper, we are concerned with finite undirected connected simple graphs. We refer to [2] for graph theoretical notation and terminology not specified here. The vertex

\footnotetext{
${ }^{*}$ The research is supported by NSFC (No. 11801487, 12061073)

†Corresponding author. E-mail: xjaxh@163.com
} 
and edge sets of $G$ are denoted by $V(G)$ and $E(G)$, respectively. We say $|V(G)|$ the order of $G$. The degree and the neighborhood of a vertex $u \in V(G)$ are denoted by $d_{G}(u)$ and $N_{G}(u)$, respectively. The length of a path between two vertices is the number of edges on that path. The distance between two vertices $u$ and $v$, denoted by $d_{G}(u, v)$, as being the length of the shortest path between them. The square of a graph $G$, denoted by $G^{2}$, is a graph with the same vertex set such that two vertices are adjacent in $G^{2}$ if and only if their distance is at most 2 in $G$.

As usual, we use $P_{n}, S_{n}, K_{n}$ to denote the path, the star, the complete graph of order $n$, respectively. A tree is called a double star $S_{p, q}$ if it is obtained from $S_{p}$ and $S_{q}$ by connecting the center of $S_{p}$ with that of $S_{q}$ via an edge. The diameter of a graph $G$, denoted by $\operatorname{diam}(G)$, is the largest distance between two vertices in $G$.

The Wiener index is a well-known distance-based topological index introduced as a structural descriptor for acyclic organic molecules [15]. It is defined as the sum of distance between all unordered pairs of vertices of a simple graph $G$, i.e.,

$$
W(G)=\sum_{u, v \in V(G)} d_{G}(u, v) .
$$

For the related results and further references, we refer to a survey [6].

The Steiner distance of a graph, introduced by Chartarand, Oellermann, Tian and Zou [4] in 1989, is a natural generalization of the distance of two vertices in a graph. For a graph $G=(V, E)$ and a set $S \subseteq V$, an $S$-Steiner tree or a Steiner tree connecting $S$ ( or simply, an $S$-tree ) is a connected subgraph $H=\left(V^{\prime}, E^{\prime}\right)$ of $G$ with $S \subseteq V^{\prime}$. Let $G$ be a connected graph of order at least 2 and let $S$ be a nonempty set of vertices of $G$. Then the Steiner distance $d(S)$ among the vertices of $S$ (or simply the distance of $S$ ) is the minimum size of a connected subgraph $H$ of $G$ such that $S \subseteq V(H)$. It is clear that $H$ must be a tree, and if $|S|=k$, then $d(S) \geq k-1$. In particular, if $S=\{u, v\}$, then $d_{G}(S)=d_{G}(u, v)$

Let $n$ and $k$ be integers such that $2 \leq k \leq n$. The Steiner $k$-eccentricity $\varepsilon_{k}(v)$ of a vertex $v$ of $G$ is defined by $\varepsilon_{k}(v)=\max \left\{d_{G}(S)|S \subseteq V(G)| S \mid,=k\right.$, and $\left.v \in S\right\}$. The Steiner $k$-radius of $G$ is $\operatorname{srad}_{k}(G)=\min \left\{\varepsilon_{k}(v) \mid v \in V(G)\right\}$, while the Steiner $k$-diameter of $G$ is $\operatorname{sdiam}_{k}(G)=\max \left\{\varepsilon_{k}(v) \mid v \in V(G)\right\}$. Note that for every connected graph $G$, $\operatorname{srad}_{2}(G)=\operatorname{rad}(G)$ and $\operatorname{sdiam}_{2}(G)=\operatorname{diam}(G)$. For more results on Steiner distance, one may see $[1,3,4,5,7,14]$. 
With respect to the concept of Steiner distance, Li, Mao, and Gutman [8] generalized the concept of Wiener index by Steiner Wiener index. For an integer $k$ with $2 \leq k \leq n-1$, the Steiner $k$-Wiener index $S W_{k}(G)$ of $G$ is the sum of Steiner $k$-distances of all subsets $S$ of $V(G)$ with $|S|=k$, that is,

$$
S W_{k}(G)=\sum_{\substack{S \subseteq V(G) \\|S|=k}} d(S) .
$$

Clearly, $S W_{2}(G)=W(G), S W_{1}(G)=0$ and $S W_{n}(G)=n-1$ for a connected graph $G$ of order $n$. For more details on Steiner Wiener index, we recommend $[8,11,12]$.

The complement $\bar{G}$ of a graph $G$ is the graph whose vertex set is $V(G)$ and whose edges are the pairs of nonadjacent vertices of $G$. In 1956, Nordhaus and Gaddum [13] proved that for a graph $G$ of order $n, 2 \sqrt{n} \leq \chi(G)+\chi(\bar{G}) \leq n+1$, where $\chi(G)$ denotes the chromatic number of $G$. Since then many research devoted to the sum on various parameters of a graph and its complement in graph theory, which are known as NordhausGaddum-type results. Mao $[9,10]$ obtained the Nordhaus-Gaddum-type results for the parameters $\operatorname{sdiam}_{k}(G)$ and the Steiner $k$-Wiener index of graphs.

Motivated by the above results, in this paper, we obtain similar results for $\operatorname{sdiam}_{3}\left(G^{2}\right)$ and the Steiner Wiener index $S W_{3}\left(G^{2}\right)$ of the square of graphs.

\section{The Steiner diameter}

Lemma 2.1. If $d_{G^{2}}(u, v)=r$ for any positive integer $r$, then $d_{G}(u, v)=2 r$ or $d_{G}(u, v)=$ $2 r-1$.

Proof. If $d_{G}(u, v) \geq 2 r+1$, then $d_{G^{2}}(u, v) \geq r+1$. If $d_{G}(u, v) \leq 2 r-2$, then $d_{G^{2}}(u, v) \leq$ $r-1$. So the result follows.

Lemma 2.2. Let $G$ be a connected graph of order $n(n \geq 3)$ and let $S \subseteq V$ be a set of vertices of $G$ with $|S|=3$. If $d_{G}(S)=2$, then $d_{G^{2}}(S)=2$; if $d_{G}(S) \geq 3$, then

$$
d_{G^{2}}(S)= \begin{cases}\left\lceil\frac{d_{G}(S)}{2}\right\rceil, & \text { if } d_{G}(S) \text { is odd }, \\ \left\lceil\frac{d_{G}(S)}{2}\right\rceil \text { or }\left\lceil\frac{d_{G}(S)}{2}\right\rceil+1, & \text { if } d_{G}(S) \text { is even. }\end{cases}
$$

Proof. Let $S=\{u, v, w\}$. If $T$ is a Steiner tree connecting $S$ in $G$ with $|E(T)|=d_{G}(S)$, then $T$ must be a path or have the form $T_{a, b, c}$ as illustrated in Figure 1(a), where $T_{a, b, c}$ is 
a tree with a vertex $z$ of degree 3 such that $T_{a, b, c}-z=P_{a} \cup P_{b} \cup P_{c}$, where $a \geq 1, b \geq 1$, $c \geq 1$ and $a+b+c \leq n-1$.

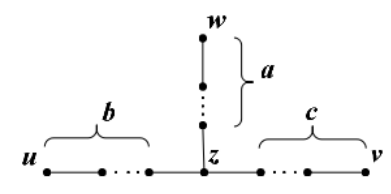

(a) $T_{a, b, c}$

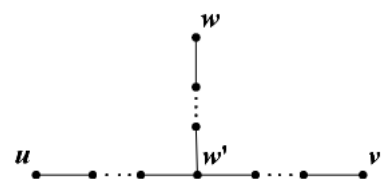

(b) $T_{a^{\prime}, b^{\prime}, c^{\prime}}$

Figure 1. Graphs for Lemma 2.2.

If $d_{G}(S)=2$, clearly $d_{G^{2}}(S)=2$. Next we assume that $d_{G}(S) \geq 3$. The structure of Steiner tree connecting $S$ in graph $G^{2}$ is shown in figure $1(\mathrm{~b})$, denote by $T_{a^{\prime}, b^{\prime}, c^{\prime}}$ and $\left|E\left(T_{a^{\prime}, b^{\prime}, c^{\prime}}\right)\right|=d_{G^{2}}(S)$. Then $d_{G^{2}}(S)=d_{G^{2}}\left(u, w^{\prime}\right)+d_{G^{2}}\left(w^{\prime}, w\right)+d_{G^{2}}\left(w^{\prime}, v\right)$. Without loss of generality, let $d_{G^{2}}\left(u, w^{\prime}\right)=t, d_{G^{2}}\left(w^{\prime}, w\right)=k$, then $d_{G^{2}}\left(w^{\prime}, v\right)=d_{G^{2}}(S)-t-k$, where $k \geq 0$. If $k=0$, then $w=w^{\prime}$. Clearly, $d_{G}(S) \leq d_{G}\left(u, w^{\prime}\right)+d_{G}\left(w^{\prime}, w\right)+d_{G}\left(w^{\prime}, v\right)$. Вy Lemma $2.1, d_{G}(S) \leq 2 t+2 k+2\left(d_{G^{2}}(S)-t-k\right)=2 d_{G^{2}}(S)$.

Case 1. All Steiner tree $T$ with $|E(T)|=d_{G}(S)$ connecting $S$ is a path in $G$.

Suppose that the path $T$ is $u \cdots w \cdots v$. We divide three subcases in terms of the parity of $d_{G}(u, w)$ and $d_{G}(w, v)$.

Case 1.1 Both $d_{G}(u, w)$ and $d_{G}(w, v)$ are even.

Let $d_{G}(u, w)=2 r, d_{G}(w, v)=2 p$. Thus $d_{G}(S)=2 r+2 p$. Then $d_{G^{2}}(S) \leq r+p=$ $\left\lceil\frac{d_{G}(S)}{2}\right\rceil$. In this case, suppose that $d_{G^{2}}(S) \leq r+p-1$, then $d_{G}(S) \leq 2 d_{G^{2}}(S) \leq 2 r+2 p-2<$ $2 r+2 p=d_{G}(S)$, a contradiction. Therefore, $d_{G^{2}}(S)=r+p=\left\lceil\frac{d_{G}(S)}{2}\right\rceil$.

Case 1.2 Both $d_{G}(u, w)$ and $d_{G}(w, v)$ are odd.

Let $d_{G}(u, w)=2 r+1, d_{G}(w, v)=2 p+1$. So, $d_{G}(S)=2 r+2 p+2$. Then $d_{G^{2}}(S) \leq$ $r+p+2=\left\lceil\frac{d_{G}(S)}{2}\right\rceil+1$. In this case, suppose that $d_{G^{2}}(S) \leq r+p+1$, then $d_{G^{2}}\left(w^{\prime}, v\right) \leq$ $r+p+1-t-k$. By Lemma $2.1, d_{G}(S) \leq 2 t+2 k+2(r+p+1-t-k)=2 r+2 p+2$. If $d_{G}(S)=2 r+2 p+2$, then $d_{G}\left(u, w^{\prime}\right)=2 t, d_{G}\left(w^{\prime}, w\right)=2 k, d_{G}\left(w^{\prime}, v\right)=2(r+p+1-t-k)$. According to Case 1, all Steiner tree $T$ connecting $S$ is a path in $G$. Therefore, $w^{\prime}=w$, then $d_{G}(u, w)=2 t$, contradict the fact that $d_{G}(u, w)=2 r+1$. Then $d_{G}(S)<2 r+2 p+2=$ $d_{G}(S)$, a contradiction. Therefore, $d_{G^{2}}(S)=r+p+2=\left\lceil\frac{d_{G}(S)}{2}\right\rceil+1$.

Case $1.3 d_{G}(u, w)$ and $d_{G}(w, v)$ have the distinct parity. 
Without loss of generality, let $d_{G}(u, w)=2 r+1, d_{G}(w, v)=2 p$. Thus $d_{G}(S)=$ $2 r+2 p+1$. Then $d_{G^{2}}(S) \leq r+p+1=\left\lceil\frac{d_{G}(S)}{2}\right\rceil$. In this case, suppose that $d_{G^{2}}(S) \leq r+p$, then $d_{G}(S) \leq 2 d_{G^{2}}(S) \leq 2 r+2 p<2 r+2 p+1=d_{G}(S)$, a contradiction. Therefore, $d_{G^{2}}(S)=r+p+1=\left\lceil\frac{d_{G}(S)}{2}\right\rceil$.

Case 2. There exists a Steiner tree $T$ with $|E(T)|=d_{G}(S)$ connecting $S$ is not a path in $G$.

We distinguish four subcases in terms of the parity of $d_{G}(u, z), d_{G}(z, v), d_{G}(z, w)$.

Case 2.1. All of $d_{G}(u, z), d_{G}(z, v), d_{G}(z, w)$ are even.

Let $d_{G}(u, z)=2 r, d_{G}(z, v)=2 q, d_{G}(z, w)=2 p$. Thus $d_{G}(S)=2 r+2 q+2 p$. Then $d_{G^{2}}(S) \leq r+q+p=\left\lceil\frac{d_{G}(S)}{2}\right\rceil$. In this case, suppose that $d_{G^{2}}(S) \leq r+q+p-1$, then $d_{G}(S) \leq 2 d_{G^{2}}(S) \leq 2 r+2 q+2 p-2<2 r+2 q+2 p=d_{G}(S)$, a contradiction. Therefore, $d_{G^{2}}(S)=r+q+p=\left\lceil\frac{d_{G}(S)}{2}\right\rceil$.

Case 2.2. One of $d_{G}(u, z), d_{G}(z, v), d_{G}(z, w)$ is odd, others are even.

Without loss of generality, let $d_{G}(u, z)=2 r+1, d_{G}(z, v)=2 q, d_{G}(z, w)=2 p$. So, $d_{G}(S)=2 r+2 q+2 p+1$. Then $d_{G^{2}}(S) \leq r+q+p+1=\left\lceil\frac{d_{G}(S)}{2}\right\rceil$. In this case, suppose that $d_{G^{2}}(S) \leq r+q+p$, then $d_{G}(S) \leq 2 d_{G^{2}}(S) \leq 2 r+2 q+2 p<2 r+2 q+2 p+1=d_{G}(S)$, a contradiction. Therefore, $d_{G^{2}}(S)=r+q+p+1=\left\lceil\frac{d_{G}(S)}{2}\right\rceil$.

Case 2.3. One of $d_{G}(u, z), d_{G}(z, v), d_{G}(z, w)$ is even, others are odd.

Without loss of generality, let $d_{G}(u, z)=2 r, d_{G}(z, v)=2 q+1, d_{G}(z, w)=2 p+1$. Thus $d_{G}(S)=2 r+2 q+2 p+2$. Then $d_{G^{2}}(S) \leq r+q+p+2=\left\lceil\frac{d_{G}(S)}{2}\right\rceil+1$. In this case, suppose that $d_{G^{2}}(S) \leq r+q+p+1$, then $d_{G^{2}}\left(w^{\prime}, v\right) \leq r+q+p+1-t-k$. By Lemma 2.1, $d_{G}(S) \leq 2 t+2 k+2(r+q+p+1-t-k)=2 r+2 q+2 p+2$. If $d_{G}(S)=2 r+2 q+2 p+2$, then $d_{G}\left(u, w^{\prime}\right)=2 t, d_{G}\left(w^{\prime}, w\right)=2 k, d_{G}\left(w^{\prime}, v\right)=2(r+q+p+1-t-k)$. In this case, $w^{\prime} \neq z$. The Steiner tree $T^{\prime}$ with $\left|E\left(T^{\prime}\right)\right|=d_{G}(S)$ connecting $S$ in $G$ is mentioned in Case 2.1. If $d_{G}(S)<2 r+2 q+2 p+2$, a contradiction. Therefore, $d_{G^{2}}(S)=r+q+p+2=\left\lceil\frac{d_{G}(S)}{2}\right\rceil+1$.

Case 2.4. All of $d_{G}(u, z), d_{G}(z, v), d_{G}(z, w)$ are odd.

Let $d_{G}(u, z)=2 r+1, d_{G}(z, v)=2 q+1, d_{G}(z, w)=2 p+1$. Thus $d_{G}(S)=2 r+2 q+2 p+$ 3. Then $d_{G^{2}}(S) \leq r+q+p+2=\left\lceil\frac{d_{G}(S)}{2}\right\rceil$. In this case, suppose that $d_{G^{2}}(S) \leq r+q+p+1$, then $d_{G}(S) \leq 2 d_{G^{2}}(S) \leq 2 r+2 q+2 p+2<2 r+2 q+2 p+3=d_{G}(S)$, a contradiction. Therefore, $d_{G^{2}}(S)=r+q+p+2=\left\lceil\frac{d_{G}(S)}{2}\right\rceil$.

So the result follows. 
By the above lemma, we conclude that $d_{P_{n}^{2}}(S)=\left\lceil\frac{d_{P_{n}}(S)}{2}\right\rceil$ or $\left\lceil\frac{d_{P_{n}}(S)}{2}\right\rceil+1$. Since $\operatorname{sdiam}_{3}\left(P_{n}\right)=n-1$, we have $\operatorname{sdiam}_{3}\left(P_{n}^{2}\right)=\left\lceil\frac{n-1}{2}\right\rceil$ or $\left\lceil\frac{n-1}{2}\right\rceil+1$. By Lemma 2.2, if $n-1$ is odd, then $\operatorname{sdiam}_{3}\left(P_{n}^{2}\right)=\left\lceil\frac{n-1}{2}\right\rceil$. Otherwise, $\operatorname{sdiam}_{3}\left(P_{n}^{2}\right)=\left\lceil\frac{n-1}{2}\right\rceil+1$. So $\operatorname{siam}_{3}\left(P_{n}^{2}\right)=\left\lceil\frac{n}{2}\right\rceil$.

Lemma 2.3. For a connected graph $G$ of $\operatorname{order} n$,

$$
2 \leq \operatorname{siam}_{3}\left(G^{2}\right) \leq\left\lceil\frac{n}{2}\right\rceil
$$

Proof. The lower bound is obvious. For the upper bound, let $T$ be a spanning tree of $G$, then $\operatorname{sdiam}_{3}(G) \leq \operatorname{sdiam}_{3}(T) \leq n-1$. Clearly, $T^{2}$ is the subgraph of $G^{2}$, thus $\operatorname{sdiam}_{3}\left(G^{2}\right) \leq \operatorname{sdiam}_{3}\left(T^{2}\right)$. There exists $S \subseteq V\left(T^{2}\right)$, such that $\operatorname{sdiam}_{3}\left(T^{2}\right)=d_{T^{2}}(S)=$ $\left\lceil\frac{d_{T}(S)}{2}\right\rceil$ or $\left\lceil\frac{d_{T}(S)}{2}\right\rceil+1$. If $d_{T}(S)<n-1$, then $\operatorname{sdiam}_{3}\left(T^{2}\right) \leq\left\lceil\frac{n}{2}\right\rceil$. From the definition of Steiner diameter and Lemma 2.2, if $d_{T}(S)=n-1$ and $n-1$ is odd, then $\operatorname{sdiam}_{3}\left(T^{2}\right)=$ $\left\lceil\frac{n-1}{2}\right\rceil=\left\lceil\frac{n}{2}\right\rceil$. Otherwise, $\operatorname{siam}_{3}\left(T^{2}\right)=\left\lceil\frac{n-1}{2}\right\rceil+1=\left\lceil\frac{n}{2}\right\rceil$.

Lemma 2.4. ([16]) Let $G$ be a connected graph with the connected complement. Then

(1) if $\operatorname{diam}(G)>3$, then $\operatorname{diam}(\bar{G})=2$,

(2) if $\operatorname{diam}(G)=3$, then $\bar{G}$ has a spanning subgraph which is a double star.

Theorem 2.5. Let $G$ be a connected graph of $n \geq 5$ with complement $\bar{G}$. Then

$$
4 \leq \operatorname{siam}_{3}\left(G^{2}\right)+\operatorname{siam}_{3}\left(\bar{G}^{2}\right) \leq \begin{cases}\left\lceil\frac{n}{2}\right\rceil+2 & \text { if } n \geq 9 \\ 6 & \text { otherwise. }\end{cases}
$$

Proof. The lower bound is obvious. Next, we prove that the right half of inequality holds. By Lemma 2.4, if $\operatorname{diam}(G)>3$, then $\operatorname{diam}(\bar{G})=2$, therefore $\bar{G}^{2} \cong K_{n}$ and $\operatorname{sdiam}_{3}\left(\bar{G}^{2}\right)=2$. By Lemma 2.3, we have $\operatorname{sdiam}_{3}\left(G^{2}\right) \leq\left\lceil\frac{n}{2}\right\rceil$. Hence,

$$
\operatorname{sdiam}_{3}\left(G^{2}\right)+\operatorname{sdiam}_{3}\left(\bar{G}^{2}\right) \leq\left\lceil\frac{n}{2}\right\rceil+2 .
$$

If $\operatorname{diam}(G)=3$, then $\operatorname{diam}(\bar{G}) \leq 3, \operatorname{diam}\left(G^{2}\right)=2, \operatorname{diam}\left(\bar{G}^{2}\right) \leq 2$, we can obtain that $\operatorname{sdiam}_{3}\left(G^{2}\right) \leq 3$ and $\operatorname{sdiam}_{3}\left(\bar{G}^{2}\right) \leq 3$, therefore,

$$
\operatorname{sdiam}_{3}\left(G^{2}\right)+\operatorname{siam}_{3}\left(\bar{G}^{2}\right) \leq 6 .
$$

If $\operatorname{diam}(G)=2$, then $\operatorname{siam}_{3}\left(G^{2}\right)=2, \operatorname{siam}_{3}\left(\bar{G}^{2}\right) \leq\left\lceil\frac{n}{2}\right\rceil$. 
Summing up the above, we conclude that

$$
4 \leq \operatorname{sdiam}_{3}\left(G^{2}\right)+\operatorname{sdiam}_{3}\left(\bar{G}^{2}\right) \leq \begin{cases}\left\lceil\frac{n}{2}\right\rceil+2, & \text { if } n \geq 9, \\ 6, & \text { otherwise }\end{cases}
$$

\section{Steiner Wiener index}

It is natural to ask, for two graph $G$ and $G_{1}$, whether it is true if $S W_{3}(G) \leq S W_{3}\left(G_{1}\right)$, $S W_{3}\left(G^{2}\right) \leq S W_{3}\left(G_{1}^{2}\right)$ in general. The answer is negative. For example, Let $G$ and $G_{1}$ be two graphs of order 7 in Fig.2. Note that $S W_{3}(G)=89<S W_{3}\left(G_{1}\right)=91$ in Fig.2, but $S W_{3}\left(G^{2}\right)=71>S W_{3}\left(G_{1}^{2}\right)=70$. For the examples of orders greater than 7 . Let $G^{\prime}$ be a graph obtained from $G$ by adding $m$ new vertices such that every vertex is only adjacent to 1,2 , and 3 . Let $G_{1}^{\prime}$ be a graph obtained from $G_{1}$ by adding $m$ new vertices such that every vertex is only adjacent to 1 and 2 . By some calculation, we have $S W_{3}\left(G^{\prime}\right)<S W_{3}\left(G_{1}^{\prime}\right)$, but $S W_{3}\left(G^{2}\right)=2\left(\begin{array}{c}m+7 \\ 3\end{array}\right)+1>S W_{3}\left(G_{1}^{2}\right)=2\left(\begin{array}{c}m+7 \\ 3\end{array}\right)$.

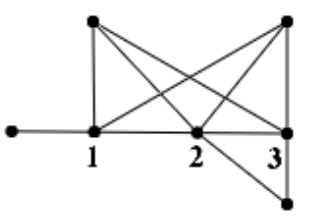

(a) $G$

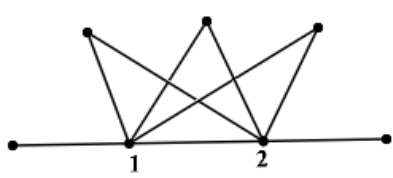

(b) $G_{1}$

Figure 2. Graphs $G$ and $G_{1}$ with $S W_{3}(G)<S W_{3}\left(G_{1}\right)$ and $S W_{3}\left(G^{2}\right)>S W_{3}\left(G_{1}^{2}\right)$.

Theorem 3.1. For any tree $T$ of order $n, S W_{3}\left(S_{n}^{2}\right) \leq S W_{3}\left(T^{2}\right) \leq S W_{3}\left(P_{n}^{2}\right)$.

Proof. Since $\operatorname{diam}\left(S_{n}\right)=2, S_{n}^{2} \cong K_{n}$. Thus $S W_{3}\left(S_{n}^{2}\right)=2\left(\begin{array}{l}n \\ 3\end{array}\right)$, and the left half of inequality holds and is best possible.

Let $T$ be a tree of order $n$. We prove that $S W_{3}\left(T^{2}\right) \leq S W_{3}\left(P_{n}^{2}\right)$ by induction on the order $n$. It is obvious that the theorem holds when $n \leq 4$. Now let $n \geq 5$. Clearly, $d_{T}(u)=1$ and $T-u$ is a tree of order $n-1$. 
By the induction hypothesis,

$$
S W_{3}\left((T-u)^{2}\right) \leq S W_{3}\left(\left(P_{n-1}^{2}\right)\right.
$$

Let $A=\left\{S: S \subseteq V\left(T^{2}\right), u \in S,|S|=3\right\}$ and $A^{\prime}=\left\{S^{\prime}: S^{\prime} \subseteq V\left(P_{n}^{2}\right), v \in S^{\prime},\left|S^{\prime}\right|=3\right\}$. We have

$$
\begin{gathered}
S W_{3}\left(P_{n}^{2}\right)=\sum_{S^{\prime} \in A^{\prime}} d_{P_{n}^{2}}\left(S^{\prime}\right)+S W_{3}\left(P_{n-1}^{2}\right), \\
S W_{3}\left(T^{2}\right)=\sum_{S \in A} d_{T^{2}}(S)+S W_{3}\left((T-u)^{2}\right) \leq \sum_{S \in A} d_{T^{2}}(S)+S W_{3}\left(P_{n-1}^{2}\right) .
\end{gathered}
$$

So, by the above inequalities (1-3), to complete the proof of $S W_{3}\left(T^{2}\right) \leq S W_{3}\left(P_{n}^{2}\right)$, it remains to show that

$$
\sum_{S \in A} d_{T^{2}}(S) \leq \sum_{S^{\prime} \in A^{\prime}} d_{P_{n}^{2}}\left(S^{\prime}\right) .
$$

This leads us to compare the value of $d_{T^{2}}(S)$ and $d_{P_{n}^{2}}\left(S^{\prime}\right)$ in term by term. We label the vertices of $P_{n}$ as $v, v_{1}, v_{2}, \ldots, v_{n-1}$, where $v v_{1} \in E\left(P_{n}\right)$ and $v_{i} v_{i+1} \in E\left(P_{n}\right)$ for each $i \in\{1, \ldots, n-2\}$. Take a longest path of $P=u u_{1} u_{2} \cdots u_{d}$ of $T$. Let $T_{i}$ be the component of $T-u_{i-1} u_{i}-u_{i} u_{i+1}$ containing $u_{i}$ for each $i \in\{1, \ldots, d-1\}$, where $u_{0}=u$. We label the vertices of $V(T) \backslash V(P)$ as $u_{d+1}, u_{d+2}, \ldots, u_{n-1}$ in terms of the following rule:

(1) subscripts of labels of vertices in $V\left(T_{i}\right) \backslash\left\{u_{i}\right\}$ is less than subscripts of labels of vertices in $V\left(T_{j}\right) \backslash\left\{u_{j}\right\}$ if $i<j$;

(2) subscripts of labels of a vertex at distance smaller from $u_{i}$ is less than subscripts of labels of the other one for any two vertices in $V\left(T_{i}\right)$ for each $i \in\{1, \ldots, d-1\}$.

We remark that if $u_{k} \in V(T) \backslash V(P)$, then $u_{k} \in V\left(T_{l}\right)$ for an integer $l \in\{1, \ldots, d-1\}$. It is easy to see that $d_{T}\left(u_{l}, u_{k}\right) \leq d_{P_{n}}\left(v_{d}, v_{k}\right)$. For $1 \leq i<j \leq n-1$, let $S_{i, j}=\left\{u, u_{i}, u_{j}\right\}$ and $S_{i, j}^{\prime}=\left\{v, v_{i}, v_{j}\right\}$.

Claim 1. $d_{T}\left(S_{i, j}\right) \leq d_{P_{n}}\left(S_{i, j}^{\prime}\right)$, with equality if and only if either $1 \leq i<j \leq d$, or $i=d<j \leq n-1$ and $d_{T}\left(u_{l}, u_{j}\right)=j-d=d_{p_{n}}\left(v_{d}, v_{j}\right)$, where $u_{j} \in T_{l}$.

Proof of Claim 1: We consider the following three cases.

Case 1. $1 \leq i<j \leq d$

It is trivial to see that $d_{T}\left(S_{i, j}\right)=d_{P_{n}}\left(S_{i, j}^{\prime}\right)$.

Case 2. $1 \leq i \leq d<j \leq n-1$

Let $u_{j} \in T_{l}$. If $1 \leq l<i \leq d$, then $d_{T}\left(S_{i, j}\right)=d_{T}\left(u, u_{i}\right)+d_{T}\left(u_{l}, u_{j}\right)$. Clearly, $d_{P_{n}}\left(S_{i, j}^{\prime}\right)=j=d+d_{p_{n}}\left(v_{d}, v_{j}\right), d_{p_{n}}\left(v_{d}, v_{j}\right) \geq d_{T}\left(u_{l}, u_{j}\right)$. Then $d_{T}\left(S_{i, j}\right) \leq d_{P_{n}}\left(S_{i, j}^{\prime}\right)$. If 
$d_{T}\left(S_{i, j}\right)=d_{P_{n}}\left(S_{i, j}^{\prime}\right)$, then $d_{T}\left(u, u_{i}\right)=d, d_{T}\left(u_{l}, u_{j}\right)=d_{p_{n}}\left(v_{d}, v_{j}\right)=j-d$. Otherwise, $d_{T}\left(S_{i, j}\right)<d_{P_{n}}\left(S_{i, j}^{\prime}\right)$.

If $1 \leq i \leq l<d$, then $d_{T}\left(S_{i, j}\right)=d_{T}\left(u, u_{l}\right)+d_{T}\left(u_{l}, u_{j}\right)<d+d_{p_{n}}\left(v_{d}, v_{j}\right)=d_{P_{n}}\left(S_{i, j}^{\prime}\right)$.

Case 3. $d<i<j \leq n-1$

Subcase $3.11 \leq m<l \leq d-1$.

Let $u_{i} \in T_{m}, u_{j} \in T_{l}$. Then $d_{T}\left(S_{i, j}\right)=d_{T}\left(u, u_{l}\right)+d_{T}\left(u_{m}, u_{i}\right)+d_{T}\left(u_{l}, u_{j}\right)$. Obviously, $d_{P_{n}}\left(S_{i, j}^{\prime}\right)=j=d+d_{p_{n}}\left(v_{d}, v_{j}\right), d_{p_{n}}\left(v_{d}, v_{j}\right) \geq d_{T}\left(u_{m}, u_{i}\right)+d_{T}\left(u_{l}, u_{j}\right)$. Thus $d_{T}\left(S_{i, j}\right)<$ $d_{P_{n}}\left(S_{i, j}^{\prime}\right)$.

Subcase $3.21 \leq m=l \leq d-1$

Set $u_{i} \in T_{l}, u_{j} \in T_{l}$, let $P_{1}$ be a shortest path of between $u_{l}$ and $u_{j}$. If $u_{i} \in V\left(P_{1}\right)$, then $d_{T}\left(S_{i, j}\right)=d\left(u, u_{l}\right)+d\left(u_{l}, u_{j}\right)<d+d_{P_{n}}\left(v_{d}, v_{j}\right)=d_{P_{n}}\left(S_{i, j}^{\prime}\right)$.

If $u_{i} \in V\left(T_{l}\right) \backslash V\left(P_{1}\right)$, let the closest vertex from $u_{i}$ to $P_{1}$ be $u_{q}$, then $d_{T}\left(S_{i, j}\right)=$ $d_{T}\left(u, u_{l}\right)+d_{T}\left(u_{l}, u_{j}\right)+d_{T}\left(u_{q}, u_{i}\right)$. Obviously, $d_{P_{n}}\left(v_{d}, v_{j}\right) \geq d_{T}\left(u_{l}, u_{j}\right)+d_{T}\left(u_{q}, u_{i}\right)$. Hence, $d_{T}\left(S_{i, j}\right)<d_{P_{n}}\left(S_{i, j}^{\prime}\right)$.

Summing up the above, we get the conclusion, as we desired.

Claim 2. If $d_{T}\left(S_{i, j}\right)<d_{P_{n}}\left(S_{i, j}^{\prime}\right)$, then $d_{T^{2}}\left(S_{i, j}\right) \leq d_{P_{n}^{2}}\left(S_{i, j}^{\prime}\right)$.

Proof: If $d_{T}\left(S_{i, j}\right)$ is odd, then $d_{T^{2}}\left(S_{i, j}\right)=\left\lceil\frac{d_{T}\left(S_{i, j}\right)}{2}\right\rceil \leq\left\lceil\frac{d_{P_{n}}\left(S_{i, j}^{\prime}\right)}{2}\right\rceil \leq d_{P_{n}^{2}}\left(S_{i, j}^{\prime}\right)$.

If $d_{T}\left(S_{i, j}\right)$ is even, then $d_{T^{2}}\left(S_{i, j}\right) \leq\left\lceil\frac{d_{T}\left(S_{i, j}\right)}{2}\right\rceil+1 \leq\left\lceil\frac{d_{P_{n}}\left(S_{i, j}^{\prime}\right)}{2}\right\rceil \leq d_{P_{n}^{2}}\left(S_{i, j}^{\prime}\right)$.

Claim 3. If $d_{T}\left(S_{i, j}\right)=d_{P_{n}}\left(S_{i, j}^{\prime}\right)$, then $d_{T^{2}}\left(S_{i, j}\right) \leq d_{P_{n}^{2}}\left(S_{i, j}^{\prime}\right)+1$, with equality, if and only if $i=d$ and $d_{T}\left(u_{l}, u_{j}\right)=j-d=d_{p_{n}}\left(v_{d}, v_{j}\right), l$ is odd, $j$ is even, $d$ is even, where $u_{j} \in T_{l}$.

Proof of Claim 3: By Claim 1, we consider the following two cases.

Case 1. $1 \leq i<j \leq d$

It is trivial to see that $d_{T^{2}}\left(S_{i, j}\right)=d_{P_{n}^{2}}\left(S_{i, j}^{\prime}\right)$.

Case 2. $i=d<j \leq n-1$ and $d_{T}\left(u_{l}, u_{j}\right)=j-d=d_{p_{n}}\left(v_{d}, v_{j}\right)$, where $u_{j} \in T_{l}$.

If $d_{T^{2}}\left(S_{d, j}\right)=\left\lceil\frac{d_{T}\left(S_{d, j}\right)}{2}\right\rceil$, then $d_{T^{2}}\left(S_{d, j}\right) \leq d_{P_{n}^{2}}\left(S_{d, j}^{\prime}\right)$. Since $d_{T}\left(S_{d, j}\right)=d_{P_{n}}\left(S_{d, j}^{\prime}\right)$, $d_{P_{n}^{2}}\left(S_{d, j}^{\prime}\right)=\left\lceil\frac{d_{P_{n}}\left(S_{d, j}^{\prime}\right)}{2}\right\rceil$ or $\left\lceil\frac{d_{P_{n}}\left(S_{d, j}^{\prime}\right)}{2}\right\rceil+1$.

If $d_{T^{2}}\left(S_{d, j}\right)=\left\lceil\frac{d_{T}\left(S_{d, j}\right)}{2}\right\rceil+1$, we divide the following three subcases by Case 2.3 in Lemma 2.2, i.e., one of $d_{T}\left(u, u_{l}\right), d_{T}\left(u_{l}, u_{d}\right), d_{T}\left(u_{l}, u_{j}\right)$ is even, others are odd.

Subcase $2.1 d_{T}\left(u, u_{l}\right)$ is even

Then $d_{T}\left(u_{l}, u_{d}\right)$ is odd, $d_{T}\left(u_{l}, u_{j}\right)=j-d=d_{p_{n}}\left(v_{d}, v_{j}\right)$ is odd. Moreover, $d_{P_{n}}\left(S_{d, j}^{\prime}\right)=$ 
$d_{P_{n}}\left(v, v_{d}\right)+d_{P_{n}}\left(v_{d}, v_{j}\right)=d_{T}\left(u, u_{d}\right)+d_{T}\left(u_{l}, u_{j}\right)$, thus $d_{P_{n}}\left(v, v_{d}\right)$ is odd and $d_{P_{n}}\left(v_{d}, v_{j}\right)$ is odd. By Case 1.2 in Lemma 2.2, $d_{P_{n}^{2}}\left(S_{d, j}^{\prime}\right)=\left\lceil\frac{d_{P_{n}}\left(S_{d, j}^{\prime}\right)}{2}\right\rceil+1$. In this case, $d_{T^{2}}\left(S_{d, j}\right)=d_{P_{n}^{2}}\left(S_{d, j}^{\prime}\right)$.

Subcase $2.2 d_{T}\left(u_{l}, u_{d}\right)$ is even

It is similar to Subcase 2.1, we can obtain $d_{T^{2}}\left(S_{d, j}\right)=d_{P_{n}^{2}}\left(S_{d, j}^{\prime}\right)$.

Subcase $2.3 d_{T}\left(u_{l}, u_{j}\right)$ is even

Then $d_{p_{n}}\left(v_{d}, v_{j}\right)=j-d=d_{T}\left(u_{l}, u_{j}\right)$ is even, $d=d_{T}\left(u, u_{l}\right)+d_{T}\left(u_{l}, u_{d}\right)$ is even, $d_{T}\left(S_{d, j}\right)=d+j-d$ is even. Thus $j$ is even. Moreover, $d_{P_{n}}\left(S_{d, j}^{\prime}\right)=d_{P_{n}}\left(v, v_{d}\right)+d_{P_{n}}\left(v_{d}, v_{j}\right)=$ $d+d_{T}\left(u_{l}, u_{j}\right)$, thus $d_{P_{n}}\left(v, v_{d}\right)$ is even and $d_{P_{n}}\left(v_{d}, v_{j}\right)$ is even. By Case 1.1 in Lemma 2.2, $d_{P_{n}^{2}}\left(S_{d, j}^{\prime}\right)=\left\lceil\frac{d_{P_{n}}\left(S_{d, j}^{\prime}\right)}{2}\right\rceil$. In this case, $d_{T^{2}}\left(S_{d, j}\right)=d_{P_{n}^{2}}\left(S_{d, j}^{\prime}\right)+1$.

Summing up the above, we get the conclusion, as we desired.

In view of Claim 3, let $A_{1}=\left\{(d, j): d_{T^{2}}\left(S_{d, j}\right)=d_{P_{n}^{2}}\left(S_{d, j}^{\prime}\right)+1, S_{d, j} \in A\right\}$, and $B_{1}=\left\{(d-1, j):(d, j) \in A_{1}\right\}$. Since $d_{T}\left(u_{l}, u_{j}\right)$ is even, we can obtain $l \neq d-1$. Clearly, $d_{T}\left(S_{d-1, j}\right)=d_{T}\left(u, u_{l}\right)+d_{T}\left(u_{l}, u_{d-1}\right)+d_{T}\left(u_{l}, u_{j}\right)=d-1+j-d=j-1$ and $d_{P_{n}}\left(S_{d-1, j}^{\prime}\right)=d_{P_{n}}\left(v, v_{d-1}\right)+d_{P_{n}}\left(v_{d-1}, v_{j}\right)=j$. By Case 2.2 and Case 1.2 in Lemma 2.2, $d_{T^{2}}\left(S_{d-1, j}\right)=\left\lceil\frac{d_{T}\left(S_{d-1, j}\right)}{2}\right\rceil=\left\lceil\frac{j-1}{2}\right\rceil, d_{P_{n}^{2}}\left(S_{d-1, j}^{\prime}\right)=\left\lceil\frac{d_{P_{n}}\left(S_{d-1, j}^{\prime}\right)}{2}\right\rceil+1=\left\lceil\frac{j}{2}\right\rceil+1$. Since $j$ is even, we have $d_{T^{2}}\left(S_{d-1, j}\right)+1=d_{P_{n}^{2}}\left(S_{d-1, j}^{\prime}\right)$. Therefore, we have

$$
\begin{aligned}
S W_{3}\left(T^{2}\right) & =\sum_{S \in A} d_{T^{2}}(S)+S W_{3}\left((T-u)^{2}\right) \leq \sum_{S \in A} d_{T^{2}}(S)+S W_{3}\left(P_{n-1}^{2}\right) \\
& =\sum_{(i, j) \in A_{1} \cup B_{1}} d_{T^{2}}\left(S_{i, j}\right)+\left(\sum_{S \in A} d_{T^{2}}(S)-\sum_{(i, j) \in A_{1} \cup B_{1}} d_{T^{2}}\left(S_{i, j}\right)\right)+S W_{3}\left(P_{n-1}^{2}\right) \\
& \leq \sum_{(i, j) \in A_{1} \cup B_{1}} d_{P_{n}^{2}}\left(S_{i, j}^{\prime}\right)+\left(\sum_{S^{\prime} \in A^{\prime}} d_{P_{n}^{2}}\left(S^{\prime}\right)-\sum_{(i, j) \in A_{1} \cup B_{1}} d_{P_{n}^{2}}\left(S_{i, j}^{\prime}\right)\right)+S W_{3}\left(P_{n-1}^{2}\right) \\
& =S W_{3}\left(P_{n}^{2}\right) .
\end{aligned}
$$

Corollary 3.2. For a connected graph $G$ of order $n, S W_{3}\left(G^{2}\right) \leq S W_{3}\left(P_{n}^{2}\right)$.

Proof. Let $T$ be a spanning tree of $G$, then $T^{2}$ is the subgraph of $G^{2}$ with vertex set $V\left(T^{2}\right)=V\left(G^{2}\right)$. For any $S, d_{G^{2}}(S) \leq d_{T^{2}}(S)$. Thus $S W_{3}\left(G^{2}\right) \leq S W_{3}\left(T^{2}\right)$. Moreover, by Theorem 3.1, $S W_{3}\left(T^{2}\right) \leq S W_{3}\left(P_{n}^{2}\right)$, the result follows.

Note that $P_{4}$ is the unique graph of order 4 whose complement is connected. Since $\overline{P_{4}} \cong P_{4}$, we have $S W_{3}\left(P_{4}^{2}\right)+S W_{3}\left({\overline{P_{4}}}^{2}\right)=2 S W_{3}\left(P_{4}^{2}\right)=16$. Next, we calculate the value 
of $S W_{3}\left(P_{n}^{2}\right)+S W_{3}\left({\overline{P_{n}}}^{2}\right)$ for $n \geq 5$. Let $P_{n}=v_{1} v_{2} \cdots v_{n}, \operatorname{sdiam}_{3}\left(P_{n}^{2}\right)=\left\lceil\frac{n}{2}\right\rceil$. For any $S \subseteq V\left(P_{n}\right)$ with $|S|=3, d_{p_{n}^{2}}(S)=m$, then $2 \leq m \leq\left\lceil\frac{n}{2}\right\rceil$. Hence, if $n$ is even,

$$
\begin{aligned}
S W_{3}\left(P_{n}^{2}\right)= & \sum_{m=2}^{\frac{n}{2}-1} m[(m-1)(n-2(m-1))+2(m-1)(n-(2 m-1)) \\
& +(m-1)(n-2 m)]+\frac{n}{2}(n-2+n-2) \\
= & \sum_{m=2}^{\frac{n}{2}-1} m(m-1)(4 n-8 m+4)+\frac{n}{2}(2 n-4) \\
= & \frac{2\left(\frac{n}{2}-1\right)\left[2 n-3\left(\frac{n}{2}-1\right)\right] \frac{n}{2}\left(\frac{n}{2}-2\right)}{3}+\frac{n}{2}(2 n-4) \\
= & \frac{n^{2}}{6}\left(\frac{n^{2}}{4}-1\right)
\end{aligned}
$$

if $n$ is odd,

$$
\begin{aligned}
S W_{3}\left(P_{n}^{2}\right)= & \sum_{m=2}^{\frac{n+1}{2}-1} m[(m-1)(n-2(m-1))+2(m-1)(n-(2 m-1)) \\
& +(m-1)(n-2 m)]+\frac{n+1}{2} \frac{n-1}{2} \\
= & \sum_{m=2}^{\frac{n+1}{2}-1} m(m-1)(4 n-8 m+4)+\frac{n+1}{2} \frac{n-1}{2} \\
= & \frac{2\left(\frac{n+1}{2}-1\right)\left[2 n-3\left(\frac{n+1}{2}-1\right)\right] \frac{n+1}{2}\left(\frac{n+1}{2}-2\right)}{3}+\frac{n+1}{2} \frac{n-1}{2} \\
= & \frac{n^{4}-4 n^{2}+3}{24}
\end{aligned}
$$

On the other hand, for $n \geq 5, \operatorname{diam}\left(\overline{P_{n}}\right)=2$ by Lemma $2.4,{\overline{P_{n}}}^{2} \cong K_{n}$ and we have $S W_{3}\left({\overline{P_{n}}}^{2}\right)=2\left(\begin{array}{l}n \\ 3\end{array}\right)$. Then

$$
S W_{3}\left(P_{n}^{2}\right)+S W_{3}\left({\overline{P_{n}}}^{2}\right)= \begin{cases}\frac{n^{2}}{6}\left(\frac{n^{2}}{4}-1\right)+2\left(\begin{array}{l}
n \\
3
\end{array}\right), & \text { if } n \text { is even, } \\
\frac{n^{4}-4 n^{2}+3}{24}+2\left(\begin{array}{l}
n \\
3
\end{array}\right), & \text { if } n \text { is odd. }\end{cases}
$$

Corollary 3.3. Let $G$ be a graph of $n \geq 5$. If $\operatorname{diam}(\bar{G})=2$ or $\operatorname{diam}(G)=2$, then $S W_{3}\left(G^{2}\right)+S W_{3}\left(\bar{G}^{2}\right) \leq S W_{3}\left(P_{n}^{2}\right)+S W_{3}\left({\overline{P_{n}}}^{2}\right)$. 
Lemma 3.4. Let $G$ be a connected graph of order $n \geq 5$ with connected complement $\bar{G}$. For any $S \subseteq V(G)$ with $|S|=3, d_{G}(S)=2$ if and only if $d_{\bar{G}}(S)>2$.

Proof. For any $S=\{u, v, w\} \subseteq V(G)$. If $d_{G}(S)=2$, then at least two elements in $\{u v, v w, u w\}$ belong to $E(G)$, at most one element in $\{u v, v w, u w\}$ belong to $E(\bar{G})$. Thus $d_{\bar{G}}(S)>2$.

Conversely, if $d_{\bar{G}}(S)>2$ and $u v, u w, v w \notin E(\bar{G})$, then the tree $T$ induced by the edges in $\{u v, u w\}$ is an $S$-Steiner tree in $G$, hence $d_{G}(S)=2$. If $d_{\bar{G}}(S)>2$ and there is an element in $\{u v, v w, u w\}$ belong to $E(\bar{G})$, without loss of generality, let $u v \in E(\bar{G})$, $u w, v w \notin E(\bar{G})$, then $u w, v w \in E(G)$, the tree $T$ induced by the edges in $\{u w, v w\}$ is an $S$-Steiner tree in $G$, namely, $d_{G}(S)=2$, as we want.

Theorem 3.5. Let $G$ be a connected graph of order $n \geq 5$ with connected complement $\bar{G}$. Then $4\left(\begin{array}{c}n \\ 3\end{array}\right) \leq S W_{3}\left(G^{2}\right)+S W_{3}\left(\bar{G}^{2}\right) \leq S W_{3}\left(P_{n}^{2}\right)+S W_{3}\left({\overline{P_{n}}}^{2}\right)$.

Proof. The lower bound is obvious. For the upper bound, from Lemma 2.4(1) and Corollary 3.3, it remains to consider the case when $\operatorname{diam}(G)=\operatorname{diam}(\bar{G})=3$. Note that $\operatorname{diam}\left(G^{2}\right)=\operatorname{diam}\left(\bar{G}^{2}\right)=2$. By Lemma 2.4(2), $G$ has a spanning subgraph which is a double star. Then $2 \leq d_{G}(S) \leq 4$ for any $S \subseteq V(G)$ with $|S|=3$. For $i=2,3$ and 4 , let $s_{i}$ be the number of all 3-element subsets of $V(G)$ with Steiner distance $i$ in $G$ and $\overline{s_{i}}$ be that for $\bar{G}$. By Lemma 3.4, $s_{2}+\overline{s_{2}}=\left(\begin{array}{l}n \\ 3\end{array}\right), s_{2}=\overline{s_{3}}+\overline{s_{4}}$ and $\overline{s_{2}}=s_{3}+s_{4}$. By Lemma 2.2 , if $d_{G}(S)=4$, then $d_{G^{2}}(S) \leq 3$. If $d_{G}(S) \leq 3$, then $d_{G^{2}}(S)=2$. Thus

$$
S W_{3}\left(G^{2}\right)+S W_{3}\left(\bar{G}^{2}\right) \leq 2 s_{2}+2 s_{3}+3 s_{4}+2 \overline{s_{2}}+2 \overline{s_{3}}+3 \overline{s_{4}}=4\left(\begin{array}{l}
n \\
3
\end{array}\right)+s_{4}+\overline{s_{4}} .
$$

By Lemma 2.4(2), let $S_{p_{1}, q_{1}}$ be a spanning subgraph of $G$ and $S_{p_{2}, q_{2}}$ be that of $\bar{G}$, where $p_{j}+q_{j}=n$ for $j=1,2$. Hence $s_{4} \leq\left(p_{1}-1\right)\left(\begin{array}{c}q_{1}-1 \\ 2\end{array}\right)+\left(\begin{array}{c}p_{1}-1 \\ 2\end{array}\right)\left(q_{1}-1\right)$ and $\overline{s_{4}} \leq\left(p_{2}-\right.$ 1) $\left(\begin{array}{c}q_{2}-1 \\ 2\end{array}\right)+\left(\begin{array}{c}p_{2}-1 \\ 2\end{array}\right)\left(q_{2}-1\right)$. Since $p_{i} \cdot q_{i} \leq\left\lfloor\frac{n^{2}}{4}\right\rfloor$ for $i=1$ and $2, s_{4} \leq \frac{\left\lfloor\frac{n^{2}}{4}\right\rfloor-n+1}{2}(n-4)$ and $\overline{s_{4}} \leq \frac{\left\lfloor\frac{n^{2}}{4}\right\rfloor-n+1}{2}(n-4)$. So

$$
S W_{3}\left(G^{2}\right)+S W_{3}\left(\bar{G}^{2}\right) \leq 4\left(\begin{array}{l}
n \\
3
\end{array}\right)+\left(\left\lfloor\frac{n^{2}}{4}\right\rfloor-n+1\right)(n-4) .
$$

One can easily check that

$$
4\left(\begin{array}{l}
n \\
3
\end{array}\right)+\left(\left\lfloor\frac{n^{2}}{4}\right\rfloor-n+1\right)(n-4) \leq \begin{cases}\frac{n^{2}}{6}\left(\frac{n^{2}}{4}-1\right)+2\left(\begin{array}{l}
n \\
3
\end{array}\right), & \text { if } n \text { is even } \\
\frac{n^{4}-4 n^{2}+3}{24}+2\left(\begin{array}{l}
n \\
3
\end{array}\right), & \text { if } n \text { is odd }\end{cases}
$$


This completes the proof.

Note that the bounds are sharp in Theorem 2.5 and Theorem 3.5. Obviously, the upper bound can be obtained on the graph $P_{n}$. To see that the lower bound is best possible, we construct a sequence of graphs. Let $G_{n}$ be a graph of order $n$, which is obtained from $C_{5}$ by replacing a vertex of $C_{5}$ by a complete graph of order $n-4$. It is easy to see that $\operatorname{diam}\left(G_{n}\right)=\operatorname{diam}\left(\overline{G_{n}}\right)=2, \operatorname{so} \operatorname{diam}\left(G_{n}^{2}\right)=\operatorname{diam}\left(\bar{G}_{n}^{2}\right)=1, \operatorname{siam}_{3}\left(G_{n}^{2}\right)+$ $\operatorname{sdiam}_{3}\left(\bar{G}_{n}^{2}\right)=4$ and $S W_{3}\left(G_{n}^{2}\right)+S W_{3}\left(\bar{G}_{n}^{2}\right)=4\left(\begin{array}{c}n \\ 3\end{array}\right)$.

\section{References}

[1] P. Ali, P. Dankelmann, S. Mukwembi, Upper bounds on the Steiner diameter of a graph, Discr. Appl. Math. 160 (2012) 1845-1850.

[2] J. A. Bondy, U. S. R. Murty, Graph Theory, Springer, New York, 2008.

[3] J. Cáceresa, A. Márquezb, M. L. Puertasa, Steiner distance and convexity in graphs, Eur. J. Combin. 29 (2008) 726-736.

[4] G. Chartrand, O. R. Oellermann, S. Tian, H. B. Zou, Steiner distance in graphs, Časopis Pest. Mat. 114 (1989) 399-410.

[5] P. Dankelmann, O. R. Oellermann, H. C. Swart, The average Steiner distance of a graph, J. Graph Theory 22 (1996) 15-22.

[6] A. Dobrynin, R. Entringer, I. Gutman, Wiener index of trees: theory and applications, Acta Appl. Math. 66 (2001) 211-249.

[7] W. Goddard, O. R. Oellermann, Distance in graphs, in: M. Dehmer (Ed.), Structural Analysis of Complex Networks, Birkhäuser, Dordrecht, 2011, pp. 49-72.

[8] X. Li, Y. Mao, I. Gutman, The Steiner Wiener index of a graph, Discuss. Math. Graph Theory 36 (2016) 455-465.

[9] Y. Mao, The Steiner diameter of a graph, Bull. Iran. Math. Soc. 43 (2017) 439-454.

[10] Y. Mao, Z. Wang, I. Gutman, H. Li, Nordhaus-Gaddum-type results for the Steiner Wiener index of graphs, Discr. Appl. Math. 219 (2017) 167-175.

[11] Y. Mao, Z. Wang, Y. Xiao, C. Ye, Steiner Wiener index and connectivity of graphs, Util. Math. 102 (2017) 51-57. 
[12] Y. Mao, Z. Wang, I. Gutman, Steiner Wiener index of graph products, Trans. Comb. 5 (2016) 39-50.

[13] E. A. Nordhaus, J. W. Gaddum, On complementary graphs, Am. Math. Mon. 63 (1956) 175-177.

[14] O. R. Oellermann, S. Tian, Steiner centers in graphs, J. Graph Theory 14 (1990) $585-597$.

[15] H. Wiener, Structural determination of paraffin boiling points, J. Am. Chem. Soc. 69 (1947) 17-20.

[16] L. Zhang, B. Wu, The Nordhaus-Gaddum-type inequalities for some chemical indices, MATCH Commun. Math. Comput. Chem. 54 (2005) 189-194. 